\title{
Neuroprotective poly(propylene imine) dendrimers with histidine- maltose shell reduce early amyloid aggregates and fibrils in Alzheimer's disease transgenic mice
}

Núria Benseny-Cases ( $\square$ nuria.benseny@uab.cat )

ALBA Synchrotron Light Source

Elena Álvarez-Marimon

Universitat Autònoma de Barcelona

\section{Ester Aso}

University of Barcelona

Margarita Carmona

University of Barcelona

Oxana Klementieva

wallenberg Neuroscience Center

Dietmar Appelhans

Leibniz Institute of Polymer Research

Isidre Ferrer

University of Barcelona

Josep Cladera

Universitat Autònoma de Barcelona

\section{Research Article}

Keywords: Amyloid plaques, Alzheimer's disease, non-invasive diagnostic techniques

Posted Date: April 7th, 2021

DOl: https://doi.org/10.21203/rs.3.rs-379309/v1

License: (c) (i) This work is licensed under a Creative Commons Attribution 4.0 International License.

Read Full License 
Neuroprotective poly(propylene imine) dendrimers with histidine-maltose shell reduce early amyloid aggregates and fibrils in Alzheimer's disease transgenic mice

Núria Benseny-Cases*a, Elena Álvarez-Marimon ${ }^{\mathrm{b}}$, Ester Aso $^{\mathrm{c}}$, Margarita Carmona ${ }^{\mathrm{c}}$, Oxana Klementieva ${ }^{\mathrm{d}}$, Dietmar Appelhans ${ }^{\mathrm{e}}$, Isidre Ferrer ${ }^{\mathrm{c}}$ and Josep Cladera ${ }^{* \mathrm{~b}}$

${ }^{a}$ ALBA Synchrotron Light Source, Carrer de la Llum 2-26, 08290 Cerdanyola del Vallès, Catalonia, Spain.

bUnitat de Biofísica i Centre d'Estudis en Biofísica, Departament de Bioquímica i de Biologia Molecular, Facultat de Medicina, Universitat Autònoma de Barcelona, 08193 Bellaterra, Catalonia, Spain.

'University of Barcelona; Biomedical Network Research Center of Neurodegenerative Diseases (CIBERNED), 08907 Hospitalet de Llobregat, Catalonia, Spain.

${ }^{d}$ Wallenberg Neuroscience Center, Lund University, BMC B11, Sölvegatan 19, 22184 Lund, Scania, Sweden.

${ }^{\text {e}}$ Leibniz Institute of Polymer Research, Dresden, Hohe Strase 6, D-01069 Dresden, Free State of Saxony, Germany.

*Corresponding authors: nbenseny@gmail.com; josep.cladera@uab.cat

Amyloid plaques composed of $A \beta$ amyloid peptides and neurofibrillary tangles are a pathological hallmark of Alzheimer Disease. In situ identification of early-stage amyloid aggregates in Alzheimer's disease is relevant for their importance as potential targets for effective drugs. Synchrotron-based infrared imaging is here used to identify early-stage oligomeric/granular aggregated amyloid species in situ in the brain of APP/PS1 transgenic mice for the first time. Also, APP/PS1 mice show fibrillary aggregates at 6 and 12 months. A significant decreased burden of early-stage aggregates and fibrillary aggregates is obtained following treatment with poly(propylene imine) dendrimers with histidine-maltose shell (a neurodegenerative protector) in 6-month-old APP/PS1 mice, thus demonstrating their putative therapeutic properties of in AD models. Identification, localization, and characterization using infrared imaging of these non-fibrillary species in the cerebral cortex at early stages of $A D$ progression in transgenic mice point to their relevance as putative pharmacological targets. No less important, early detection of these structures may be useful in the search for markers for non-invasive diagnostic techniques. 
Alzheimer's Disease (AD) is characterized by the presence of senile plaques mainly composed of $\mathrm{A} \beta$ peptides and neurofibrillary tangles resulting from aberrant intraneuronal deposition of phosphorylated tau species. However, recent experimental evidence supports the idea that amyloid fibrils are not necessarily toxic whereas toxic species relevant to the onset and progression of the disease are found among a diverse population of on- and off-pathway nonfibrillary intermediates. Although some of these non-fibrillary amyloids and their toxicity have been described in vitro, and different oligomeric species have been isolated from affected brain homogenates ${ }^{1-3}$, the challenge remains regarding their identification in vivo and the time at which they form, presumably long before the appearance of neurological deficits. Whereas powerful imaging techniques such as MRI and PET are being used to detect the presence of fibrillary amyloids, and in some cases, oligomeric species have been targeted, ${ }^{4-9}$ the detection of earlier non-fibrillary aggregates and their structural characterization, which would facilitate the design of effective drugs, remain elusive.

Infrared microscopy ( $\mu$-FTIR) has been used in the last decade for the in situ study of amyloid deposits. ${ }^{10-14}$ In the present work, we have used synchrotron-based $\mu$-FTIR to describe the early formation of non-fibrillar amyloid aggregates in brains of APP/PS1 transgenic mice ${ }^{15-18}$ at 3, 6, and 12 months and to evaluate the effect of poly(propylene imine) dendrimers with histidinemaltose shell (G4-His-Mal dendrimers) ${ }^{19}$ as anti-neurodegenerative agents in mice.

\section{RESULTS AND DISCUSSION}

Fourier Transform Infrared (FTIR) spectra were measured in the cerebral cortex of 3-, 6-, and 12month-old APP/PS1 transgenic mice, age-matched wild type (WT) mice and 6-month-old APP/PS1 mice treated with -G4-His-Mal dendrimers. Supplementary Figs. S1 and S2 provide two examples of the regions measured in the animal's cortex and how the detected amyloid deposits co-localize with a positive anti-amyloid antibody label in a contiguous slide of the tissue. The scores graph and the corresponding loadings of the Principal Component Analysis (PCA) of the whole set of data is shown in supplementary Fig. S3. In order to facilitate data visualization and interpretation the score plot of the whole data set has been broken down and the resulting graphs are shown in Figures 1 and 5 in this section. Representing the principal component 1(PC1) versus the principal component 3 (PC3) has given a score graph in which two set of outliers (points corresponding to spectra outside the region in which the WT spectra appear) are more clearly resolved than in the PC1vs PC2 plot (data not shown). According to the loadings graph, the outliers on the PC1 axis differ from the WT spectra in a main feature centred at $1628 \mathrm{~cm}^{-1}$, characteristic of peptide/protein fibrillary aggregation. The PC3 loadings show two features centred at 1620 and $1695 \mathrm{~cm}^{-1}$, characteristic of non-fibrillary/amorphous/granular (non-fibrillar $\beta$-sheet) structures. ${ }^{20-22}$

\section{Oligomeric/Granular (O/G) and fibrillary amyloid aggregates in APP/PS1 mice at 3, 6 and 12 months of age.}

Fig. 1 shows the score plot of the PCA of the amide I region for 3-, 6-, and 12-month-old APP/PS1 represented together with WT data scores. Since, according to the loadings graph (supplementary Fig. S1) the typical wavenumber for intermolecular fibrillary $\beta$-sheet is the dominant feature of PC1, PC1 can be used to classify the spectra as a function of fibrillary amyloid content. In the same way, being PC3 indicative of non-fibrillary $\beta$-sheet structures, PC3 values can be used to classify the spectra depending on the presence of oligomeric/granullar $\beta$-sheet content. We have done so by segmenting the data points outside the area corresponding to the WT points in two different groups: outliers in the direction of the negative PC1 (segmented in orange in Fig.1); and outliers in the direction of the positive PC3 (segmented in blue in Figure 1). 

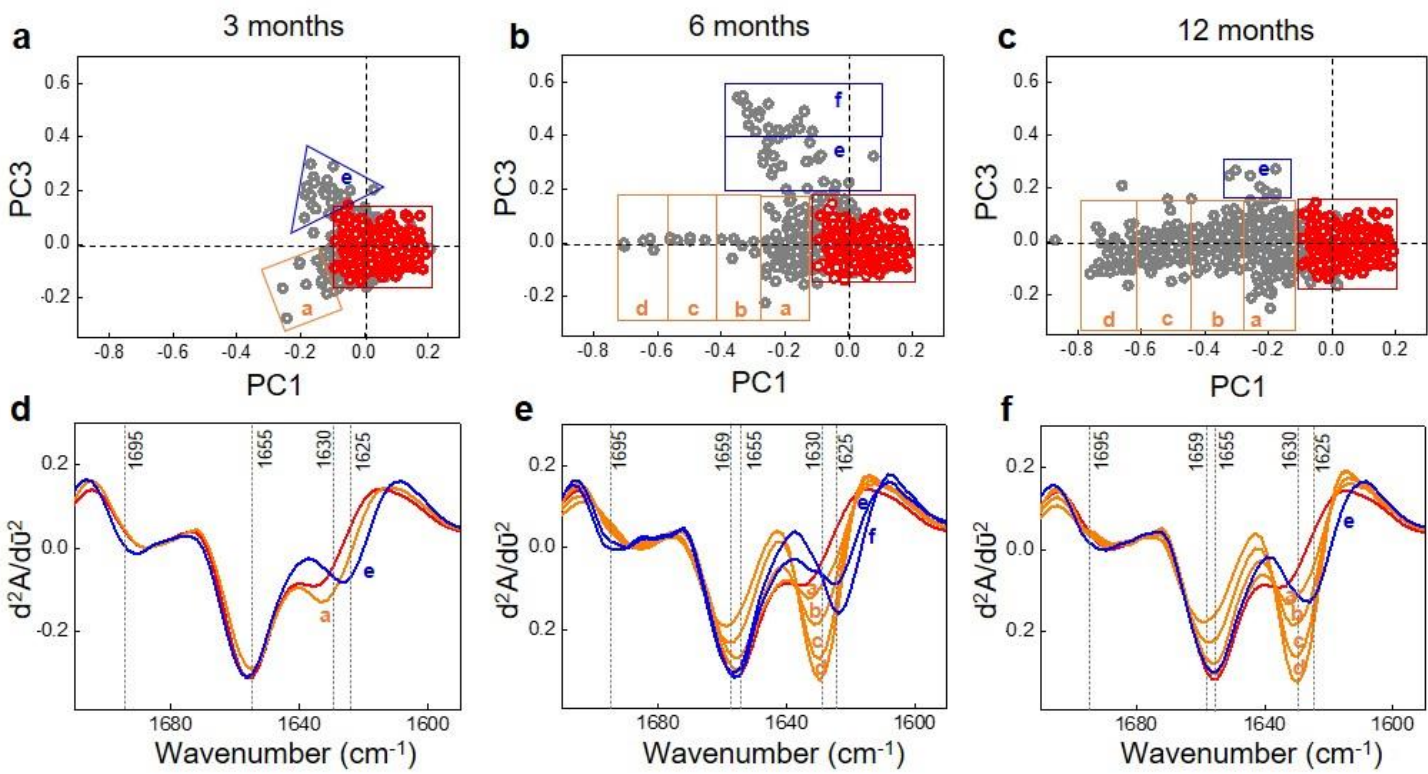

Figure 1. Principal component analysis (PCA) score plots of infrared spectra from brain samples of WT mice and APP/PS1 mice 3 months (a), 6 months (b) and 12 months (c) old. WT data is shown in red and APP data in grey. The loadings corresponding to the PCA scores are shown in Fig. S1 together with the PCA scores of the whole set of data using in this study (APP/PS1 + treated with G4-His-Mal dendrimer). The bottom row shows the normalized second derivative mean spectra of the amide I region calculated from the individual spectra in each outlier segment (a-f) on the PCA scores, for APP/PS1 mice 3 months (d), 6 months (e) and 12 months (f) old. Colour code: each average derivative spectrum has the same colour as the corresponding coloured segment in which the outlier spectra appear in the top row. In orange, segments a-d in the direction of the negative PC1 axis; in blue, segments e,f in the direction of the positive PC3 component; in red, the average spectrum of the WT data points.

In order to further characterize the structural differences between the different groups of outliers the mean of the FTIR spectrum of all data points in each segment in figure 1 was calculated and compared with the average spectra of the WT points. The derivative spectra corresponding to the PC1 segments (a-d), depicted in orange in figure 1 (bottom panels) show a $\beta$-sheet fibrillary aggregation band at $1630 \mathrm{~cm}^{-1}$ (consistent with the $1628 \mathrm{~cm}^{-1}$ feature detected in the PC1 loadings in figure S3), which intensity increases as the $\mathrm{PC1}$ value becomes more negative, together with a shift of the minimum at $1655 \mathrm{~cm}^{-1}$ towards $1659 \mathrm{~cm}^{-1}$. Fibrillary structures can therefore be characterized by the presence of these two features in the second derivative spectra: a band at $1630 \mathrm{~cm}^{-1}$ and an increase in intensity at $1659 \mathrm{~cm}^{-1}$. The derivative spectra corresponding to the PC3 segments (e,f), depicted in blue, show a band at $1625 \mathrm{~cm}^{-1}$ (this band represents a shift with respect to the $1630 \mathrm{~cm}^{-1}$ band of the fibrillary structures and is compatible with the shifted 1620 $\mathrm{cm}^{-1}$ feature in the PC3 loadings in figure S3) and a band centred at $1695 \mathrm{~cm}^{-1}$ (the same wavelength observed in the PC3 loadings), both characteristic of a non-fibrillar $\beta$-sheet structure. Comparison of these spectral features with the FTIR (second derivative) spectra of known aggregated species generated in vitro, shown in Fig. 2, permits to clearly identify the aggregated species detected in APP/PS1 mice brains as oligomeric/granular aggregates $(\mathrm{O} / \mathrm{G}, \beta$ structures of non-fibrillary nature, with a high frequency component, characteristic of non-fibrillary $\beta$ sheets) and fibrillary aggregates (F). The biophysical analysis of these in vitro aggregates is displayed in Fig. S4, showing the morphology of the fibrils and the non-fibrillary aggregates together with the ThT fluorescence kinetics, which is clearly more intense in the presence of fibrils. Such infrared spectral features for non-fibrillary and fibrillary amyloid structures have also been reported in previous in vitro studies ${ }^{20-22}$. It is worth emphasizing that the spectra depicted in Fig. 2 correspond 
to the amyloid peptide aggregated under incubation conditions that lead to the formation of either fibrillary aggregates (neutral $\mathrm{pH}$ ) or amorphous/granular aggregates $(\mathrm{pH} 5.5)$. The spectral features of the amorphous aggregated species at $\mathrm{pH} 5.5$ turn out to be very similar to those reported by Sarroukh et al.$^{21}$ for oligomeric $A \beta$ (both $A \beta(1-40)$ and $A \beta(1-42)$ ). Our own data together with previous studies show that the spectral features of fibrils and oligomeric/amorphous/granular aggregates are very similar for both $A \beta(1-40)$ and $A \beta(1-42)$. This means that infrared spectra cannot be used as a tool to distinguish between oligomers and amorphous aggregates nor to differentiate the aggregates formed by $A \beta(1-40)$ from the ones formed by $A \beta(1-42)$. However, it clearly differentiates fibrillary from non-fibrillary aggregates.
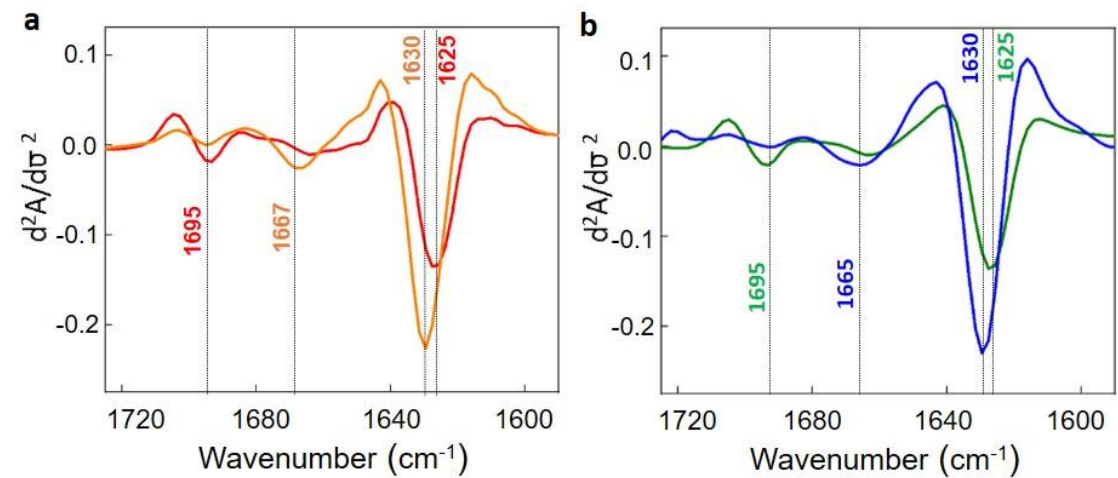

Figure 2. Average second derivative spectra of the amide I region of (a) $A \beta(1-40)$ and (b) $A \beta(1-42)$ amyloid aggregates generated in vitro: fibrils (orange and blue respectively) generated at $\mathrm{pH} 7.4$ and $\mathrm{A} \beta$ granular non-fibrillary aggregates (red and green respectively) generated at $\mathrm{pH}$ 5.5. The absorbance spectra were normalized for the amide I area prior to derivation.

The correspondence between the distinct spectroscopic features and the fibrillary/non-fibrillary structures is proven by the TEM images shown in Fig. S4. In relation to the aggregated structures at $\mathrm{pH} 5.5$, although the TEM images do not permit to deduce any structural detail other than the amorphous/granular aspect, it is clear that the aggregates are non-fibrillary, and besides the large amorphous clumps individual round dots, compatible with big oligomeric structures are normally distinguished. In our previous studies ${ }^{20}$ with $\mathrm{A} \beta(1-40)$ we showed that granular (amorphous) aggregates formed at $\mathrm{pH} 5.5$ could be transformed into fibrils by increasing the $\mathrm{pH}$ to 7.4 and that this transformation followed the typical sigmoidal kinetics. For this reason, we speculated with the possibility that these granular aggregates could be a source of oligomeric forms given the right conditions (i.e. an increase of the $\mathrm{pH}$ ). For all these reasons we refer to the amorphous aggregates of granular aspect with the same spectroscopic characteristics as oligomeric structures as $\mathrm{O} / \mathrm{G}$ aggregates. $\mathrm{A} \beta(1-40) \mathrm{O} / \mathrm{G}$ aggregates are known to be toxic to neuroblastoma cells in culture. ${ }^{20}$ Toxicity assays with $\mathrm{A} \beta(1-42)$ have shown that this peptide incubated at $\mathrm{pH} 5.5$ is as well toxic (data not shown).

It can be concluded from Fig. 1 that these two types of aggregated structures (fibrillary and $\mathrm{O} / \mathrm{G}$ non-fibrillary aggregates) start forming in APP/PS1 mice brains at 3 months of age, both types do clearly increase at 6 months and at 12 months a further increase of the fibrillary structures and a decrease of the $\mathrm{O} / \mathrm{G}$ aggregates is detected.

These results, showing the evolution of both types of amyloid aggregates with mice age, can be formulated as well in terms of the differences in the values of the spectroscopic ratios reported in Fig. 3. For all the ratios, the main band of the WT spectra at $1655 \mathrm{~cm}^{-1}$ was taken as the reference band against which the intensity of the characteristic bands of the different aggregated species was measured. The ratios $\mathrm{A}_{1630} / \mathrm{A}_{1655}$ and $\mathrm{A}_{1659} / \mathrm{A}_{1655}$ were taken as the spectroscopic signature of 
fibrillary aggregates, and the ratios $\mathrm{A}_{1695} / \mathrm{A}_{1655}$ and $\mathrm{A}_{1625} / \mathrm{A}_{1655}$ as the spectroscopic signature of the $\mathrm{O} / \mathrm{G}$ aggregates.

Experimental points with values of the ratios at $\mathrm{A}_{1630} / \mathrm{A}_{1655}$ and $\mathrm{A}_{1659} / \mathrm{A}_{1655}$ (fibrillary structures) higher than the values calculated for the WT are clearly more abundant at 6 and 12 months and their abundance increase with age (Fig. 3 a, b).

Fig. 3 (c, d), shows that the abundance of $\mathrm{O} / \mathrm{G}$ non-fibrillary structures is significantly increased in APP/PS1 compared to WT (experimental points with a value of the ratios $\mathrm{A}_{1695} / \mathrm{A}_{1655}$ and $\mathrm{A}_{1625} / \mathrm{A}_{1655}$ higher than the values calculated for the wild type), and they are more abundant at 3 and 6 months than at 12 months.
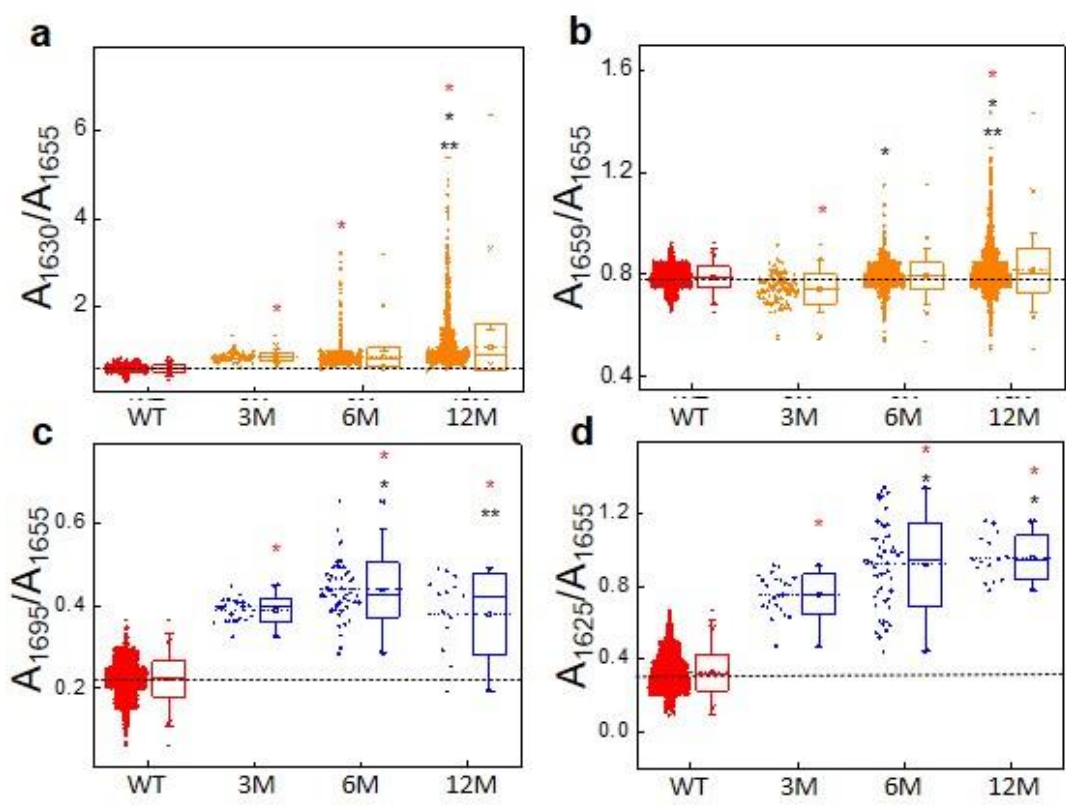

Figure 3. Box plot graphics depicting the absorbance ratio values corresponding to the two different types of aggregated species identified at 3, 6, and 12 months in APP/PS1 and WT mice. Amyloid fibrils (a, b: the ratios $A_{1630} / A_{1655}$ and $A_{1659} / A_{1655}$ are the two spectral features of fibrillary structures as explained in the Results and Discussion section) and Oligomeric/granular aggregates (c, d: the ratios $A_{1695} / A_{1655}$ and $\mathrm{A}_{1625} / \mathrm{A}_{1655}$ are the two spectral features of non-fibrillary aggregated structures as explained in the Results and Discussion section). Ratios are calculated from the second derivative spectra of the outliers in each segment (a-f) in the PCA shown in Fig. 1. Boxplot shows the median (center line), interquartile range (IQR) (box); whiskers represent the most extreme data located no more than 1.5xIQR from the edge of the box, and outliers are the points outside this range. T-test is used for comparison with WT (red*), 12 month mice $(*)$, and between 3 and 6 month mice $(* *)$ at $\mathrm{p}<0.002$.

Since $\mu$-FTIR makes possible the representation of the spatial distribution of the detected chemical species, the different types of amyloid aggregates (fibrillary, non-fibrillary $\mathrm{O} / \mathrm{G}$ ) can be visualized in the form of infrared maps using the corresponding absorbance ratios, as illustrated in Fig. 4. In the figure, the infrared maps are shown together with the corresponding image obtained in the visible region of a contiguous brain slide labelled with a fluorescent amyloid antibody. A more detailed illustration of the co-localization of the infrared regions in which the infrared spectra have been measured, with the anti-amyloid positively labelled plaques using a contiguous tissue slide is given in supplementary Figs. S1 and S2. 


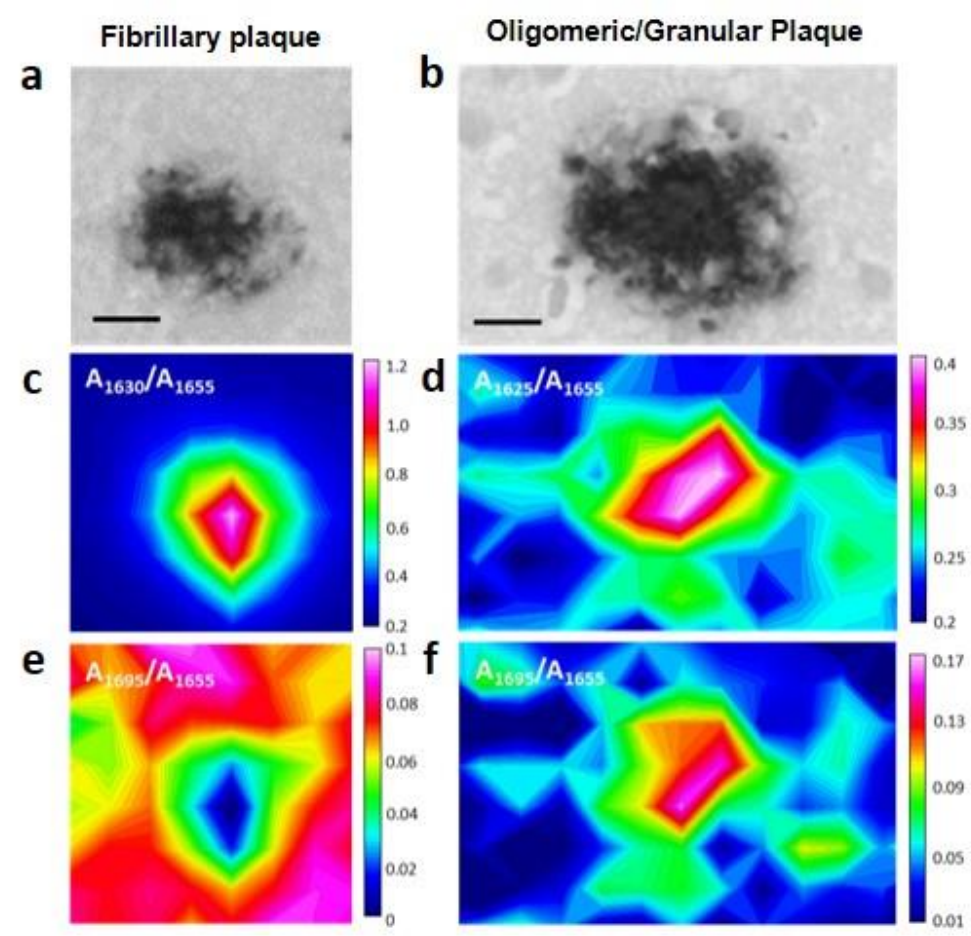

Figure 4. Representative infrared and visible maps of a fibrillary plaque (left panels) and an Oligomeric/Granullar plaque (right panels). (Top row) Images in the visible region of the plaque labeled with an antiamyloid antibody in a contiguous brain slide; (Midle row) absorbance ratios corresponding to fibrillary $\beta$-sheet amyloid aggregation; (Bottom row) absorbance ratios corresponding to antiparallel $\beta$ sheet aggregation distribution. Ratios were calculated from the second derivative spectra. Scale bar $=10$ $\mu \mathrm{m}$.

\section{Effect of G4-His-Mal dendrimers on amyloid aggregation in APP/PS1 mice brains.}

G4-His-Mal dendrimers are biocompatible globular branched polymers containing maltose and histidine on their surface with capacity to protect $\mathrm{AD}$ transgenic mice from memory impairment. ${ }^{19}$ Using $\mu$-FTIR we have analysed the effect of G4-His-Mal dendrimers on the aggregated species in the brains of 6-month-old APP/PS1 mice. For these experiments 6-month-old APP/PS1 mice were chosen as they showed significant memory impairment as revealed in the V-maze. ${ }^{18}$

Recently, our group has proven the potential of G4-His-Mal dendrimers to protect APP/PS1 mice from memory impairment and to reduce the aggregation of $A \beta 40$ in vitro ${ }^{19}$. Although previous analysis using OC antibodies seemed to show an increase in A $\beta$ fibrils ${ }^{19}, \mu$-FTIR clearly shows (Fig. 5) that the treatment of APP/PS1 with G4-His-Mal dendrimers results in reduced formation of early $(\mathrm{O} / \mathrm{G})$ amyloid aggregates and that the amount of fibrillary $\beta$-sheet is decreased in number and intensity. For the $\mathrm{O} / \mathrm{G}$ aggregates the presence of non-fibrillary $\beta$-sheet-bands is significantly reduced compared to APP/PS1 mice of the same age.

In a previous paper, Klementieva et al. ${ }^{23}$ described the formation of pre-plaque non-fibrillary amyloid structures in $\operatorname{Tg} 19959$ transgenic mice. In the present study, we have confirmed the presence of non-fibrillary structures in APP/PS1 mice and have characterized the structure of these aggregates in more detail. Moreover, we show that treatment with G4-His-Mal dendrimers reduces fibrillary aggregation and, importantly, the formation of non-fibrillary $(\mathrm{O} / \mathrm{G})$ aggregates. 
a

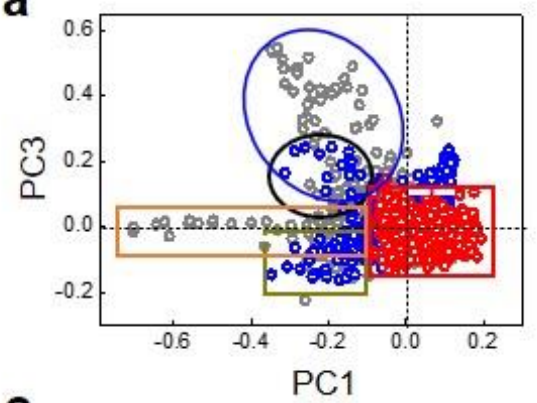

C

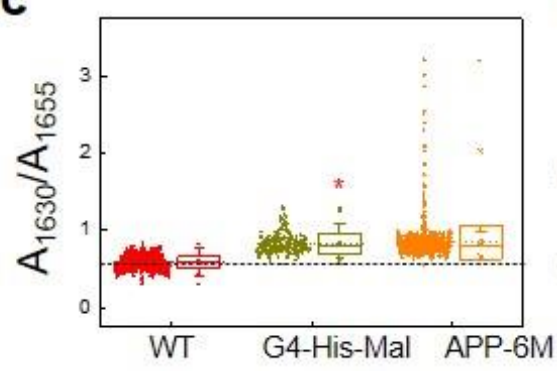

b

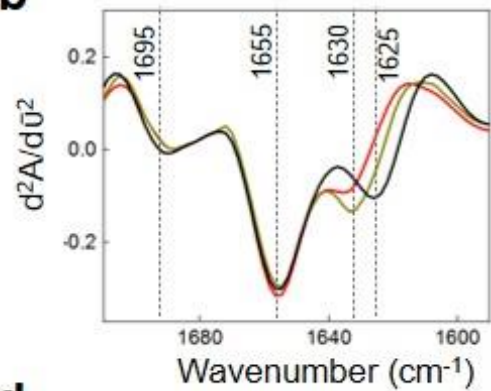

d

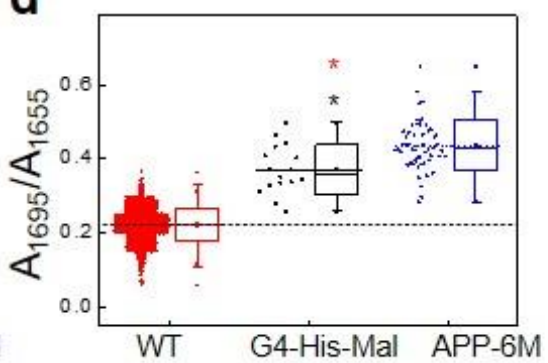

Figure 5. (a) Scores of the Principal component analysis (PCA) using the second derivative of the amide I region of infrared spectra showing data from WT mice, APP/PS1 mice 6 months old and APP/PS1 6 months old treated with G4-His-Mal dendrimers. WT data is shown in red, APP/PS1 data in grey and APP/PS1 treated with G4-His-Mal dendrimers in blue. The loadings corresponding to the PCA scores are depicted in Fig. S1 together with the PCA scores of the whole set of data used in this study; (b) normalized mean second derivative of the amide I region calculated from the individual spectra of the data points within the colour circle/rectangle in the outlier regions of the PCA scores. The bottom row shows the box plot graphics depicting the absorbance ratio values corresponding to the two different types of aggregated species calculated from the second derivative spectra of the data points within the colour circle/rectangle in the outlier regions of the PCA scores. Fibrillary aggregates are shown in (c) and oligomeric/granular aggregates in (d). Boxplot shows the median (center line), interquartile range (IQR) (box); whiskers represent the most extreme data located no more than 1.5xIQR from the edge of the box, and outliers are the points outside this range. T-test is used for comparison with WT $\left(\right.$ red $\left.^{*}\right)$ and 6 months mice $(*$ black) at $\mathrm{p}<0.002$. Colour code: each set of point in the box plots has the same colour as the circle with which the data points from which the ratios have been marked in the scores plot.

\section{CONCLUSION}

Altogether, our results show that non-fibrillary aggregated species $(\mathrm{O} / \mathrm{G})$ are abundant in the cerebral cortex at an early stage of disease progression in APP/PS1 transgenic mice. Moreover, the levels of such non-fibrillary structures (and the aggregation level in general) are severely reduced in APP/PS1 mice treated with the spherical dendritic scaffold of H-bond- and ionic interaction-active G4-His-Mal dendrimers.

These non-fibrillary aggregated forms have previously been described in a number of in vitro studies including the formation of synthetic $\mathrm{A} \beta(1-40)$ amorphous/granular toxic aggregates at $\mathrm{pH}$ 5.5 and in the presence of metal ions and of unordered structures in amyloid aggregates, the occurrence of oligomeric species containing non-fibrillary $\beta$ structures, and the purification of different types of oligomers such as $\mathrm{A} \beta * 56$ from brain homogenates. ${ }^{20-22,24-26}$

Identification, localization, and further characterization of these non-fibrillary species in the cerebral cortex at early stages of $\mathrm{AD}$ progression in transgenic mice point to their relevance as putative pharmacological targets. Successful decreased deposition of these species following treatment with G4-His-Mal dendrimers buoys further research in this line. No less important, 
early detection of these structures may be useful in the search for markers for non-invasive diagnostic techniques.

\section{MATERIALS AND METHODS}

\section{Animals}

All animal procedures were conducted according to the European Union directive 2010/63/EU and approved by the ethics committee of the University of Barcelona. APP/PS1 transgenic mice express a chimeric mouse/human APP (Mo/HuAPP695swe: APP Swedish mutation) and a mutant human presenilin 1 (PS1-dE9); the included Swedish mutation (K595N/M596L) elevates the amount of $\beta$-amyloid, and the mutant PS1 allele accelerates the $\beta$-amyloid deposition rate as well as exacerbating pathological severity ${ }^{18}$. APP/PS1 transgenic mice and wild-type (WT) littermates were used for the experiments. The human mutated forms APPswe and PS1dE9 were developed by co-injection of the two transgene constructs $[\mathrm{Mo} / \mathrm{Hu}$ "humanized" chimeric APP695 harbouring the Swedish (K594M/N595L) mutation and exon-9-deleted PS1] delivered by mouse prion promoter into pronuclei with a single genomic insertion site, resulting in the two transgenes being transmitted as a single Mendelian locus. To homogenize the genetic background of mice, the first heterozygous generation was bred for at least 15 generations on a C57BL6J background, with selection for the double mutant transgenes at each generation. Afterward, heterozygote/WT mating produced WT and double-transgenic APP/PS1 littermates for subsequent experiments.

To screen for the presence of the transgenes, genomic DNA was isolated from $1 \mathrm{~cm}$ tail clips and genotyped with the polymerase chain reaction (PCR) technique using the PCR conditions proposed by Jackson Laboratory. For the experiment, animals were maintained under standard animal housing conditions in a 12-h dark-light cycle with free access to food and water. The experiments were carried out in compliance with the ARRIVE guidelines.

\section{Preparation of mice brain tissue for synchrotron FTIR mapping}

WT and APP/PS1 mice aged 3, 6, and 12 months ( $\mathrm{n}=3-4$ per time point) were deeply anaesthetized by intraperitoneal injection $(0.2 \mathrm{~mL} / 10 \mathrm{~g}$ body weight $)$ with a mixture of ketamine $(100 \mathrm{mg} / \mathrm{kg})$ and xylazine $(20 \mathrm{mg} / \mathrm{kg})$ prior to intracardiac perfusion of $4 \%$ paraformaldehyde delivered with a peristaltic pump at $19 \mathrm{~mL} /$ minute for 5 minutes. Brains were removed and postfixed overnight at $4{ }^{\circ} \mathrm{C}$ in the same fixative solution. Tissue samples were embedded in paraffin, and coronal sections $8 \mu \mathrm{m}$ thick were cut on a microtome and mounted on $\mathrm{CaF}_{2}$ windows (Crystran, U.K).

\section{Synthesis of Poly (propylene imine) maltose-histidine (G4His-Mal) dendrimers}

Synthesis of G4HisMal was performed as in Aso et al. ${ }^{19}$. Briefly, poly(propylene imine) dendrimers of the 4th generation were modified with His (G4His), and then G4His was modified with maltose (G4HisMal). The whole conversion process was carried out in argon protection atmosphere. The structure of G4HisMal dendrimers is depicted in supplementary Fig. S5.

\section{G4His-Mal dendrimers treatment}

The experiments were carried out on male APP/PS1 and wild-type mice. At the age of 3 months, animals were randomly divided as follows: 7 transgenic and 6 WT mice received $5 \mu \mathrm{g}$ per day G4HisMal; 5 transgenic and 6 WT mice received the same volume of PBS $(5 \mu \mathrm{L})$ until animals reached the age of 6 months.

\section{Preparation of $A \beta(1-40)$ aggregates in vitro}

A $\beta(1-40)$ peptide [DAEFRHDSGYEVHHQKLVFFAEDVGSNKGAIIGLMVGGVV] was purchased from JPT (Germany) with $\mathrm{Cl}^{-}$as a counterion. The peptide $(500 \mu \mathrm{M})$ was dissolved in $10 \mathrm{mM}$ PBS buffer with $0.04 \% \mathrm{NH}_{3}$ at $\mathrm{pH} 12$ (pH adjusted using $\mathrm{NaOH}$ ) and sonicated for $30 \mathrm{~s}$ to ensure that it was in monomeric condition as described in Benseny-Cases et al. ${ }^{20}$ The stock 
solutions were kept at $-80^{\circ} \mathrm{C}$ until use. The stock solutions were diluted at $200 \mu \mathrm{M}$ in PBS buffer and the $\mathrm{pH}$ was adjusted to $\mathrm{pH} 7.4$ (to trigger amyloid fibril formation) and to $\mathrm{pH} 5.5$ (to trigger amyloid amorphous aggregates formation) using $\mathrm{HCl}$ and were then incubated overnight and mixed at $200 \mathrm{rpm}$ and $37^{\circ} \mathrm{C}$. After incubation, in vitro aggregates were dried directly on $\mathrm{CaF}_{2}$ windows.

\section{SR-FTIR microspectroscopy and data acquisition}

SR- $\mu$ FTIR was performed at the MIRAS beamline at ALBA synchrotron (Catalonia, Spain), using a Hyperion 3000 Microscope that was equipped with a $36 \times$ magnification objective coupled to a Vertex 70 spectrometer (Bruker). The measuring range was $650-4000 \mathrm{~cm}^{-1}$ and the spectrum collection was carried out in transmission mode at $4 \mathrm{~cm}^{-1}$ resolution, $10 \mu \mathrm{m} \times 10 \mu \mathrm{m}$ aperture dimensions, and co-added from 64-128 scans. Zero filling was performed with fast Fourier transform (FFT) so that in the final spectra there was one point every $2 \mathrm{~cm}^{-1}$. Background spectra were collected from a clean area of the $\mathrm{CaF}_{2}$ window every 10 min. Mercury-cadmium-telluride (MCT) detector was used and the microscope and spectrometer were continuously purged with nitrogen gas.

\section{Fourier Transform Infrared (FTIR) spectrum analysis}

Fourier transform infrared (FTIR) spectra from the different maps, and the independent spectra of amyloid aggregates were analyzed with Opus 7.5 (Bruker) software. Atmospheric compensation was applied to the spectra to remove water vapour and $\mathrm{CO}_{2}$ contributions. The spectra exhibiting a low signal-to-noise ratio were eliminated and concave rubberband baseline corrected (RBC) in the range of 3100-1400 $\mathrm{cm}^{-1}$ using 64 baseline points and 5 iterations. An example of one set of spectra after and before rubberband corrections is displayed in supplementary Figure S6. Unscrambler X software (CAMO) Software (Oslo, Norway) was used to perform PCA in the data set as in Benseny-Cases et al. ${ }^{11}$ Briefly, PCA analysis was applied on the second derivative of the spectra calculated using a Savitsky-Golay algorithm with a thirteen point filter and a polynomial order of 3 . Unit vector normalization was applied after secondary derivation for PCA analysis.

Principal components (PCs) were calculated using the Unscrambler X software (CAMO) Software was also used for the normalized average spectra and the second derivative spectrum calculation. Ratios were calculated over the following peaks representing different protein secondary structures in the Amide I region: $1695 \mathrm{~cm}^{-1} / 1655 \mathrm{~cm}^{-1}$ associated to $1625 \mathrm{~cm}^{-1} / 1655$ $\mathrm{cm}^{-1}$ for the non-fibrillary (oligomeric, granular aggregates) $\beta$-sheet structures (noted as $\mathrm{A}_{1695} / \mathrm{A}_{1655}$ and $\mathrm{A}_{1625} / \mathrm{A}_{655}$ ), $1630 \mathrm{~cm}^{-1} / 1655 \mathrm{~cm}^{-1}$ (noted as $\mathrm{A}_{1630} / \mathrm{A}_{1655}$ ) and $1659 \mathrm{~cm}^{-1} / 1655 \mathrm{~cm}^{-1}$ (noted as $A_{1659} / A_{1655}$ ) for the fibrillary $\beta$-sheet structures. Origin 9.1 software was used for the ratio calculation and graphical representation.

\section{Immunohistochemistry}

Consecutive $4 \mu \mathrm{m}$ slices of the tissue samples were embedded in paraffin used for the $\mu$ FTIR analyses were cut and placed in polyLys treated glass slides. Samples were de-waxed by submerging the samples on xylene ( $3 \times 8 \mathrm{~min})$ and samples were hydrate in $100 \%, 95 \%$, and $75 \%$ ethanol for $2 \times 4$ minutes each. De-waxed sections were incubated with $98 \%$ formic acid (3 minutes) and samples were boiled in citrate buffer (20 minutes) to enhance antigenicity. Then, the endogenous peroxidases were blocked with Peroxidase-Blocking solution (Dako, Denmark) (15 minutes) and then incubated at $4{ }^{\circ} \mathrm{C}$ overnight with the primary antibodies against $\beta$-amyloid (Clone 6F/3D)(Dako). Sections were subsequently rinsed and incubated with biotinylated goat anti-mouse \& rabbit secondary antibody (Dako), followed by EnVision+ System Peroxidase (Dako) and finally with the chromogen diaminobenzidine and $\mathrm{H}_{2} \mathrm{O}_{2}$. Sections were lightly counterstained with hematoxylin. After staining, the sections were dehydrated and cover-slipped for microscopic observation. ${ }^{18}$ 


\section{AUTHOR CONTRIBUTION}

Authors made substantial contributions to conception and design (N. B-C; J.C.; D. A.; O. K.; I. F.; E. A.), and/or acquisition of data (N. B-C; J.C.; E. A-M; O. K.; E. A.; M. C.), and/or analysis and interpretation ((N. B-C; J.C.; D. A.; O. K.; I. F.; E. A.), as well as redaction of the draft and final manuscript ((N. B-C; J.C.; D. A.; O. K.; I. F.; E. A.; E. A-M).

\section{ACKNOWLEDGMENTS}

This study was funded by grant SAF2017-844-R to J.C. from the Spanish Ministerio de Ciencia, Innovación y Universidades, programa RETOS 2017, and by an ALBA Synchrotron in-house project IH18MIRAS grant to N.B-C. The study received funding from "la Caixa" Foundation under the agreement LCF/PR/HR19/52160007 to IF. We thank CERCA Programme/Generalitat de Catalunya for institutional support. We thank T. Yohannan for editorial help and R. Zinke (IPF Dresden/Germany) for the synthesis of G4-His-Mal dendrimers.

\section{COMPETING INTERESTS}

The authors declare no competing interests.

\section{REFERENCES}

1. Selkoe, D.J. \& Hardy, J. The amyloid hypothesis of Alzheimer's disease at 25 years. EMBO Mol. Med. 8, 595-608; 10.15252/emmm.201606210 (2016).

2. Hayden, E.Y. \& Teplow, D.B. Amyloid $\beta$-protein oligomers and Alzheimer's disease. Alzheimers Res. Ther. 5, 60-71; 10.1186/alzrt226 (2013).

3. K.L. Viola \& W.L. Klein. Amyloid $\beta$ oligomers in Alzheimer's disease pathogenesis, treatment, and diagnosis. Acta Neuropathol. 129, 183-206; 10.1007/s00401-015-1386-3 (2015).

4. Femminella, G.D. et.al. Imaging and Molecular Mechanisms of Alzheimer's Disease: A Review. Int. J. Mol. Sci. 19, 3702-3725; 10.3390/ijms19123702 (2018).

5. Dudeffant, C.M. et.al. Contrast-enhanced MR microscopy of amyloid plaques in five mouse models of amyloidosis and in human Alzheimer's disease brains. Sci. Rep. 7, 4955-49969; 10.1038/s41598-017-05285-1 (2017).

6. Viola, K.L et.al. Towards non-invasive diagnostic imaging of early-stage Alzheimer's disease. Nat. Nanotechnol. 10, 91-98; 10.1038/nnano.2014.254 (2015).

7. Viola, K.L. \& Klein, W.L. Amyloid $\beta$ oligomers in Alzheimer's disease pathogenesis, treatment, and diagnosis. Acta Neuropathol. 129, 183-206; 10.1007/s00401-015-1386-3 (2015).

8. Nordberg, A., Rinne, J.O., Kadir, A. \& Langström, B. The use of PET in Alzheimer disease. Nat. Rev. Neurol. 6, 78-87; 10.1038/nrneurol.2009.217 (2010).

9. Frisoni, G.B., Fox, N.C., Jack, Jr. C. R., Scheltens, P. \& Thompson, P.M. The clinical use of structural MRI in Alzheimer disease Nat. Rev. Neurol. 6, 67-77; 10.1038/nrneurol.2009.215 (2010).

10. Benseny-Cases, N., Klementieva, O., Cotte, M., Ferrer, I. \& Cladera, J. Microspectroscopy $(\mu$ FTIR) Reveals Co-localization of Lipid Oxidation and Amyloid Plaques in Human Alzheimer Disease Brains. Anal. Chem. 86, 12047-12054; 10.1021/ac502667b (2014).

11. Benseny-Cases, N. et.al. Synchrotron-Based Fourier Transform Infrared Microspectroscopy ( $\mu$ FTIR) Study on the Effect of Alzheimer's A $\beta$ Amorphous and Fibrillar Aggregates on PC12 Cells. Anal. Chem. 90, 2772-2779; 10.1021/acs.analchem.7b04818. (2018).

12. Choo, L.P. et.al. In situ characterization of beta-amyloid in Alzheimer's diseased tissue by synchrotron Fourier transform infrared microspectroscopy. Byophys. J. 71, 1672-1679; 10.1016/S0006-3495(96)79411-0 (1996).

13. Miller, L.M. \& Dumas, P. Chemical imaging of biological tissue with synchrotron infrared light. Biochim. Bipohys. Acta. 1758, 846-857; 10.1016/j.bbamem.2006.04.010 (2006).

14. Miller, L.M., Bourassa, M. W. \& Smith, R.J. FTIR spectroscopic imaging of protein aggregation in living cells. Biochim. Bipohys. Acta 1828, 2339-2346; 10.1016/j.bbamem.2013.01.014 (2013). 
15. Borchelt, D.R et.al. Familial Alzheimer's disease-linked presenilin 1 variants elevate Abeta1-42/1-40 ratio in vitro and in vivo. Neuron 17, 1005-1013; 10.1016/s0896-6273(00)80230$\underline{5}(1996)$.

16. Blanchard, V. R. et.al. Time sequence of maturation of dystrophic neurites associated with Abeta deposits in APP/PS1 transgenic mice. Exp Neurol. 184, 247-263; 10.1016/s0014-

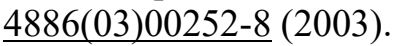

17. Savonenko, A. et.al Episodic-like memory deficits in the APPswe/PS1dE9 mouse model of Alzheimer's disease: relationships to beta-amyloid deposition and neurotransmitter abnormalities. Neurobiol. Dis. 18, 602-617; 10.1016/j.nbd.2004.10.022 (2005).

18. Aso, E. et.al. Amyloid generation and dysfunctional immunoproteasome activation with disease progression in animal model of familial Alzheimer's disease. Brain Pathol. 22, 636-653; 10.1111/j.1750-3639.2011.00560.x (2012).

19. Aso, E et.al. Poly(propylene imine) dendrimers with histidine-maltose shell as novel type of nanoparticles for synapse and memory protection. Nanomedicine 17, 198-209; 10.1016/j.nano.2019.01.010 (2019).

20. Benseny-Cases, N., Klementieva, O., Maly, J. \& Cladera, J. Granular non-fibrillar aggregates and toxicity in Alzheimer's disease. Curr. Alzheimer Res. 9, 962-971; 10.2174/156720512803251129 (2012).

21. Sarroukh, R.,et al.ATR-FTIR: a "rejuvenated" tool to investigate amyloid proteins. Biochim. Biophys. Acta 1828, 2328-2338; 10.1016/j.bbamem.2013.04.012 (2013).

22. Cerf, E. et.al. Antiparallel beta-sheet: a signature structure of the oligomeric amyloid betapeptide. Biochem. J. 421, 415-423. 10.1042/BJ20090379 (2009).

23. Klementieva, O. et.al. Pre-plaque conformational changes in Alzheimer's disease-linked A $\beta$ and APP. Nat. Commun. 8, 14726-14734; 10.1038/ncomms14726 (2017).

24. Jiang, D. et al.Aggregation pathways of the amyloid $\beta(1-42)$ peptide depend on its colloidal stability and ordered $\beta$-sheet stacking. Langmuir, 28, 12711-12721; 10.1021/1a3021436 (2012).

25. Breydo, L. et.al. Structural differences between amyloid beta oligomers. Biochem. Biophys. Res. Commun. 477, 700-705; 10.1016/j.bbrc.2016.06.122 (2016).

26. Lesne, S. et.al. A specific amyloid-beta protein assembly in the brain impairs memory. Nature 16, 352-357; 10.1038/nature04533 (2006). 


\section{Figures}
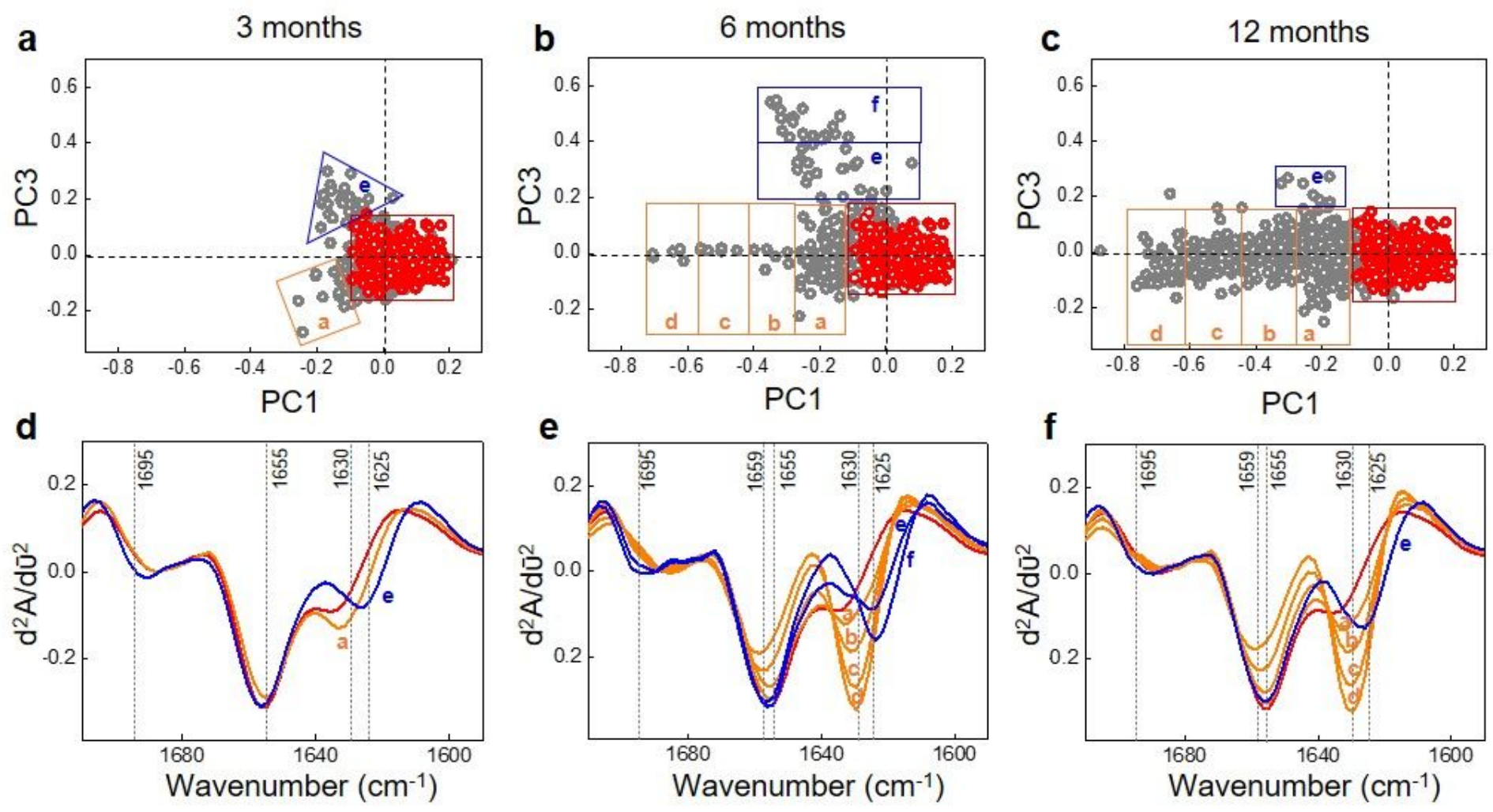

Figure 1

Principal component analysis (PCA) score plots of infrared spectra from brain samples of WT mice and APP/PS1 mice 3 months (a), 6 months (b) and 12 months (c) old. WT data is shown in red and APP data in grey. The loadings corresponding to the PCA scores are shown in Fig. S1 together with the PCA scores of the whole set of data using in this study (APP/PS1 + treated with G4-His-Mal dendrimer). The bottom row shows the normalized second derivative mean spectra of the amide I region calculated from the individual spectra in each outlier segment (a-f) on the PCA scores, for APP/PS1 mice 3 months (d), 6 months (e) and 12 months (f) old. Colour code: each average derivative spectrum has the same colour as the corresponding coloured segment in which the outlier spectra appear in the top row. In orange, segments a-d in the direction of the negative PC1 axis; in blue, segments e, $f$ in the direction of the positive PC3 component; in red, the average spectrum of the WT data points. 

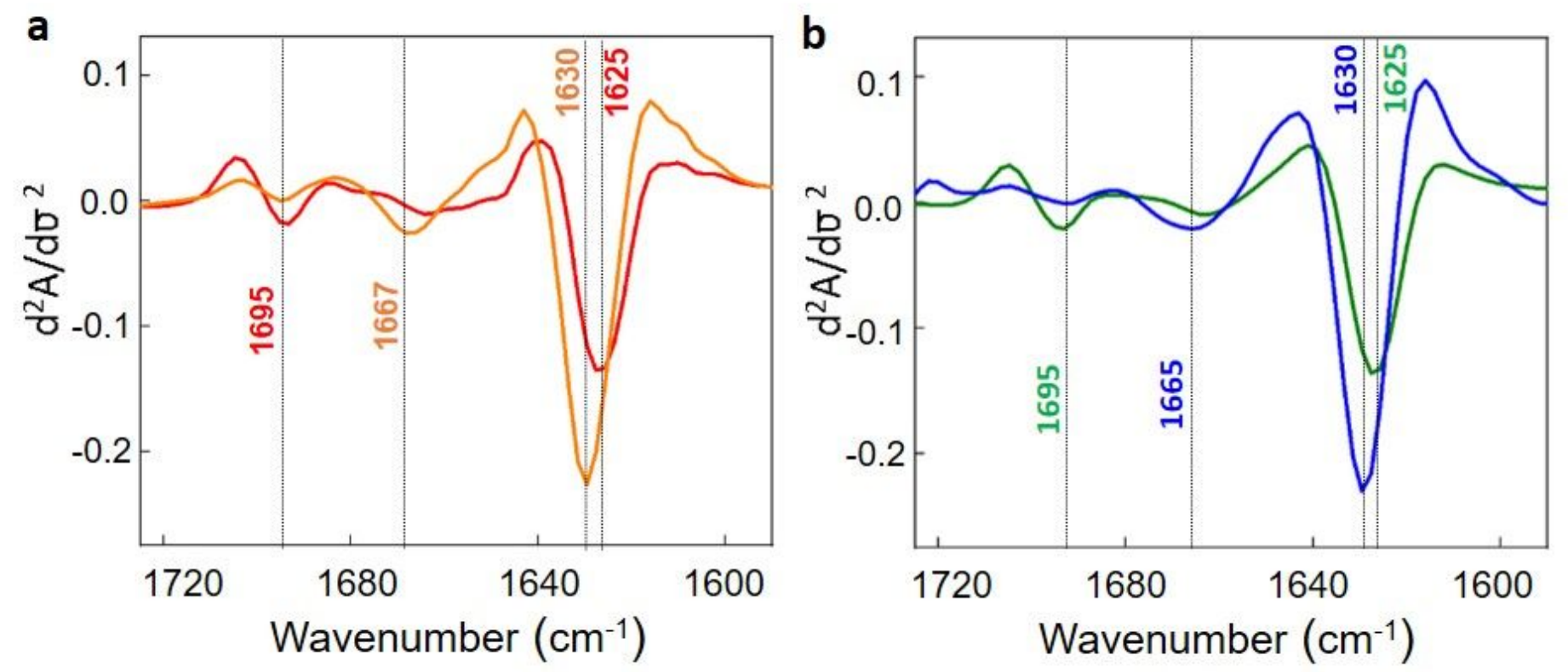

Figure 2

Average second derivative spectra of the amide I region of (a) $A \beta(1-40)$ and (b) $A \beta(1-42)$ amyloid aggregates generated in vitro: fibrils (orange and blue respectively) generated at $\mathrm{pH} 7.4$ and $\mathrm{A} \beta$ granular non-fibrillary aggregates (red and green respectively) generated at $\mathrm{pH}$ 5.5. The absorbance spectra were normalized for the amide I area prior to derivation.
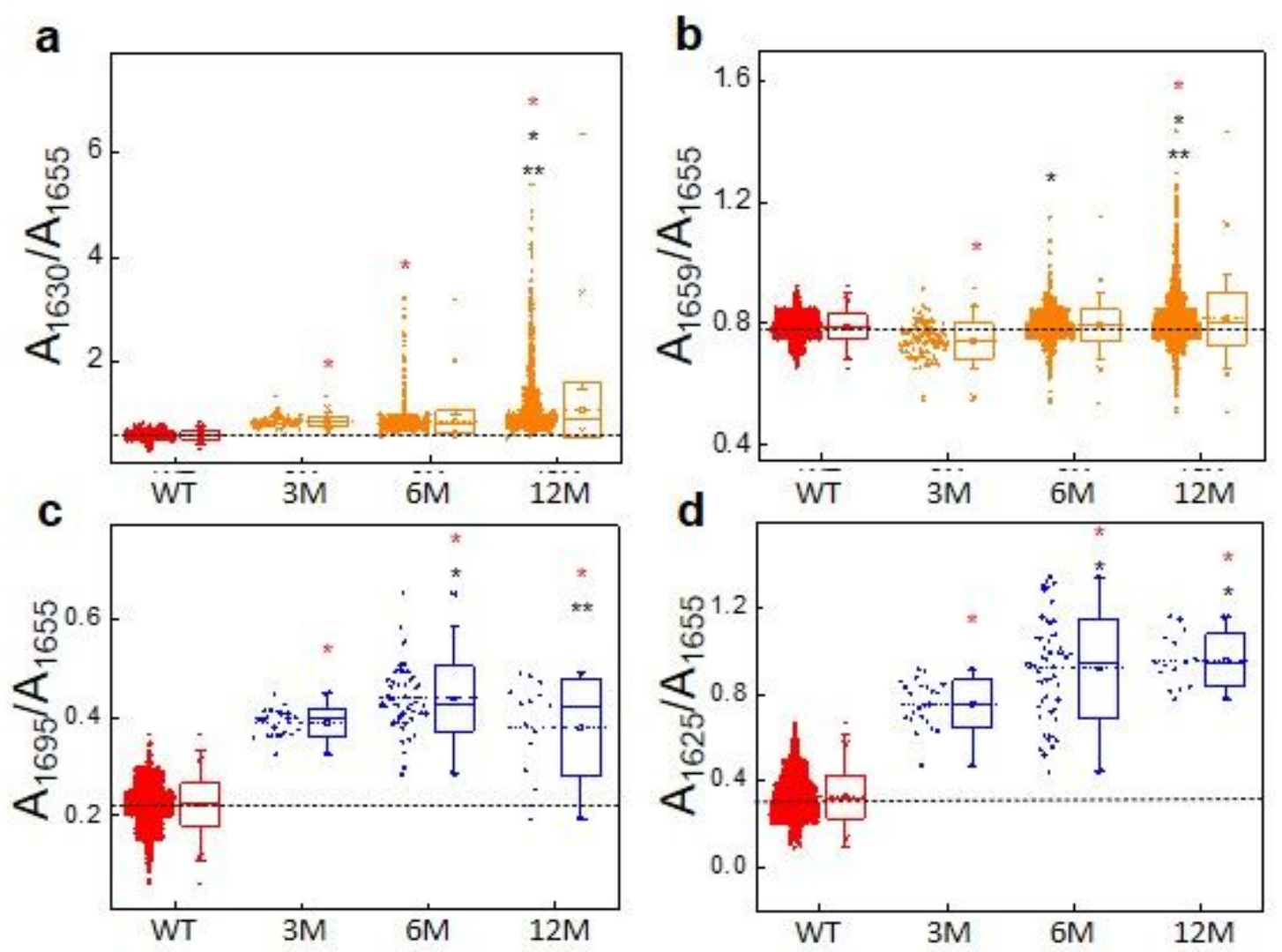


\section{Figure 3}

Box plot graphics depicting the absorbance ratio values corresponding to the two different types of aggregated species identified at 3, 6, and 12 months in APP/PS1 and WT mice. Amyloid fibrils ( $a$, b: the ratios A1630/A1655 and A1659/A1655 are the two spectral features of fibrillary structures as explained in the Results and Discussion section) and Oligomeric/granular aggregates (c, d: the ratios A1695/A1655 and A1625/A1655 are the two spectral features of non-fibrillary aggregated structures as explained in the Results and Discussion section). Ratios are calculated from the second derivative spectra of the outliers in each segment (a-f) in the PCA shown in Fig. 1. Boxplot shows the median (center line), interquartile range (IQR) (box); whiskers represent the most extreme data located no more than 1.5xIQR from the edge of the box, and outliers are the points outside this range. T-test is used for comparison with WT (red*), 12 month mice $\left({ }^{*}\right)$, and between 3 and 6 month mice $\left.{ }^{* *}\right)$ at $p<0.002$.

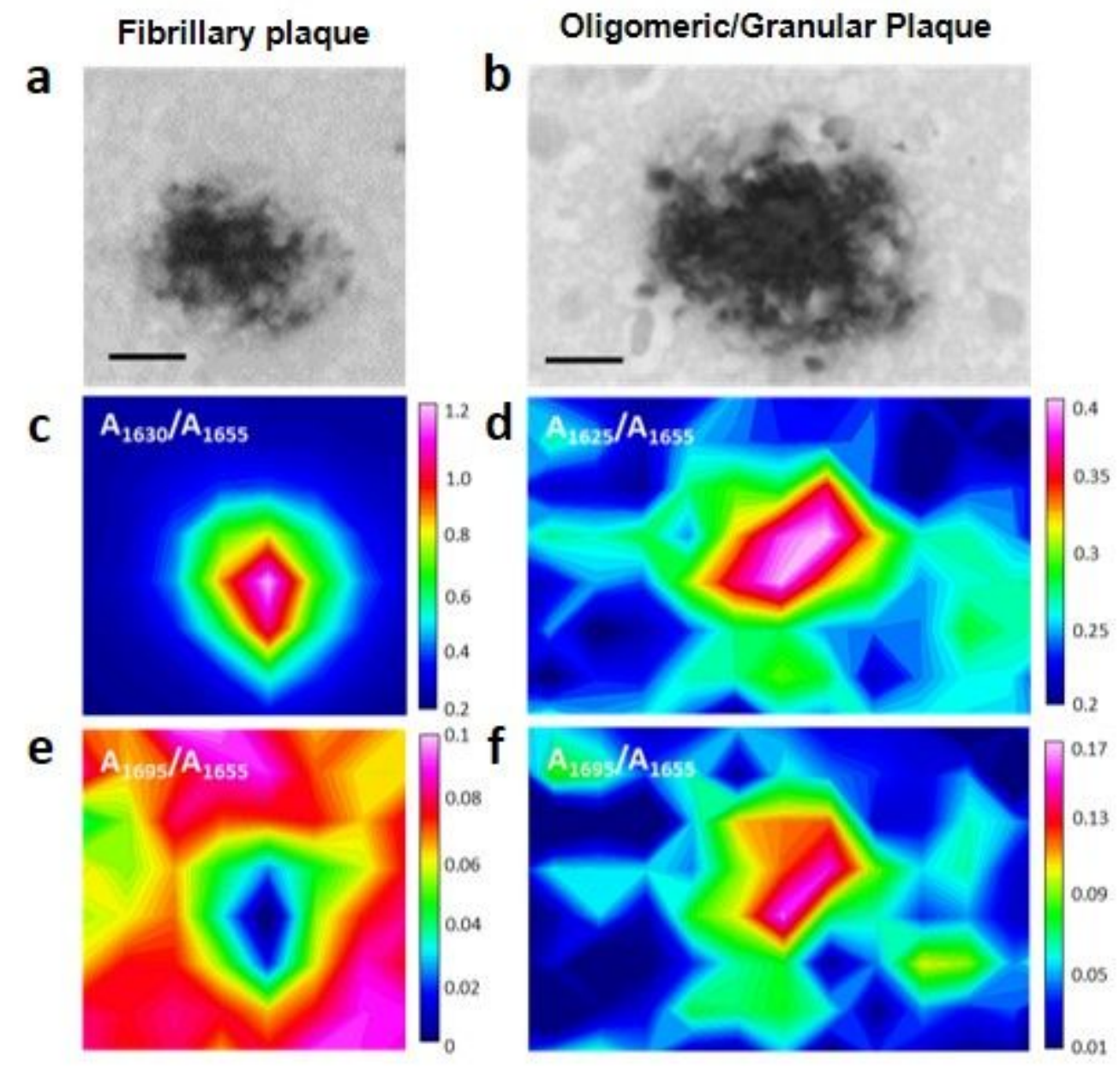

\section{Figure 4}

Representative infrared and visible maps of a fibrillary plaque (left panels) and an Oligomeric/Granullar plaque (right panels). (Top row) Images in the visible region of the plaque labeled with an antiamyloid antibody in a contiguous brain slide; (Midle row) absorbance ratios corresponding to fibrillary $\beta$-sheet amyloid aggregation; (Bottom row) absorbance ratios corresponding to antiparallel $\beta$-sheet aggregation distribution. Ratios were calculated from the second derivative spectra. Scale bar $=10 \mu \mathrm{m}$. 

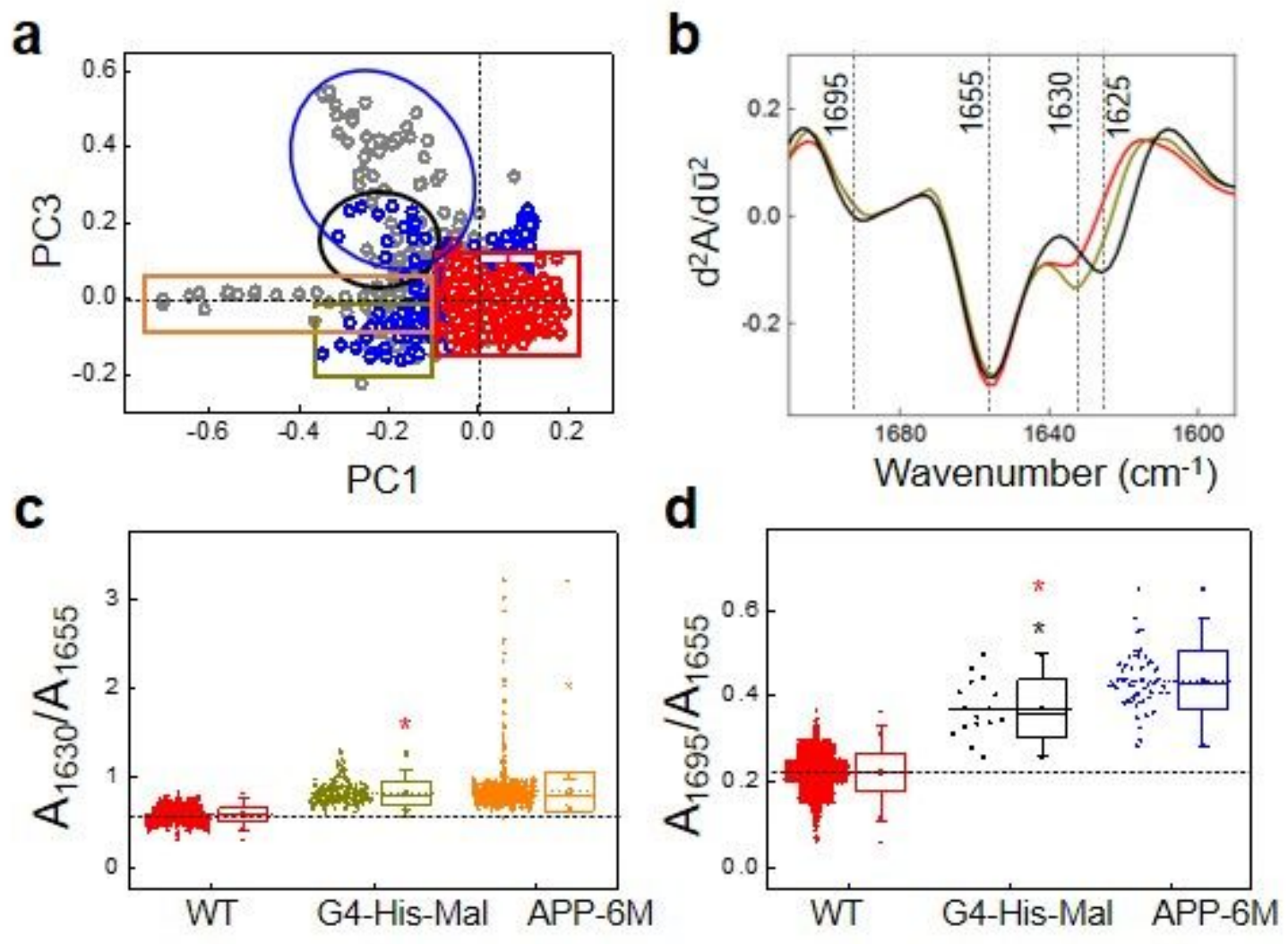

Figure 5

(a) Scores of the Principal component analysis (PCA) using the second derivative of the amide I region of infrared spectra showing data from WT mice, APP/PS1 mice 6 months old and APP/PS1 6 months old treated with G4-His-Mal dendrimers. WT data is shown in red, APP/PS1 data in grey and APP/PS1 treated with G4-His-Mal dendrimers in blue. The loadings corresponding to the PCA scores are depicted in Fig. S1 together with the PCA scores of the whole set of data used in this study; (b) normalized mean second derivative of the amide I region calculated from the individual spectra of the data points within the colour circle/rectangle in the outlier regions of the PCA scores. The bottom row shows the box plot graphics depicting the absorbance ratio values corresponding to the two different types of aggregated species calculated from the second derivative spectra of the data points within the colour circle/rectangle in the outlier regions of the PCA scores. Fibrillary aggregates are shown in (c) and oligomeric/granular aggregates in (d). Boxplot shows the median (center line), interquartile range (IQR) (box); whiskers represent the most extreme data located no more than 1.5xIQR from the edge of the box, and outliers are the points outside this range. T-test is used for comparison with WT (red*) and 6 months mice (*black) at $p<0.002$. Colour code: each set of point in the box plots has the same colour as the circle with which the data points from which the ratios have been marked in the scores plot.

\section{Supplementary Files}

This is a list of supplementary files associated with this preprint. Click to download. 
- SIscirepBensenyCases.pdf 